“C 2007 IEEE. Personal use of this material is permitted. Permission from IEEE must be obtained for all other uses, in any current or future media, including reprinting/republishing this material for advertising or promotional purposes, creating new collective works, for resale or redistribution to servers or lists, or reuse of any copyrighted component of this work in other works." 


\title{
Robust Stabilization of LPV Systems with Structured Uncertainty using Minimax Controllers
}

\author{
Li Li and Valery A. Ugrinovskii
}

\begin{abstract}
This paper addresses a robust control scheduling scheme for uncertain linear parameter-varying systems with structured uncertainty. A gain-scheduled controller is proposed which employs a set of minimax optimal robust controllers and incorporates an interpolation rule to achieve continuity of the controller gain over a range of operating conditions. Novel weighted time-domain integral quadratic constraints are introduced to assist in the derivation of the controller. The key idea of the interpolation for the structured uncertainty case is to transform the parameterized algebraic Riccati inequalities into equivalent linear matrix inequalities. For every fixed value of the system parameter, the proposed controller guarantees robust stability and a certain bound on the worst-case performance of the corresponding uncertain closed loop system. Furthermore, a bound on the rate of parameter variations is obtained under which the closed loop LPV system is robustly stable. To obtain the proposed controller, a set of semi-definite programming problems are introduced; this enables an efficient numerical solution to the problem under consideration.
\end{abstract}

\section{INTRODUCTION}

The problem of analytical gain-scheduling has been of increasing interest since 1990s [1], [2]. Recently, the interest in this technique has significantly increased owing to the progress in the area of parameter dependent linear matrix inequalities (LMIs) [3], [4]. Following [5], the LMI approach to gain-scheduling relies on converting the underlying control problem into a set of $H_{\infty}$ control problems parametrized by the scheduling parameter and then converting these problems into an infinite array of LMIs. To overcome the infinite dimensionality of the resulting LMI problem, relaxation techniques are considered [4]; to achieve relaxation of the LMIs to a finite dimensional problem, an LFT dependency structure of the system on the scheduling parameters [6], polynomial representations [7] or rational approximations [8] are often needed. The technical assumption of convexity of the set of models is another essential requirement of this approach [6], [7], [9], which may result in more conservative performance.

Different from the class of uncertain systems considered in the mentioned references, we consider Linear Parameter Varying (LPV) systems with structured uncertainty shown in Figure 1 in which $\Delta_{j}$ are non-stationary operators

$$
w_{j}(t)=\Delta_{j}\left(t,\left.z_{j}(\cdot)\right|_{0} ^{t}\right), \quad j=1, \ldots, n,
$$

This work is supported by the Australian Research Council and the University of New South Wales.

L. Li is with National ICT Australia, Department of Electrical and Electronic Engineering, The University of Melbourne, VIC, 3010, Australia li.lidee.unimelb.edu.au.

V. A. Ugrinovskii is with School of ITEE, University of New South Wales at the Australian Defence Force Academy, Canberra ACT 2600, Australia, v.ougrinovskiladfa.edu.au.

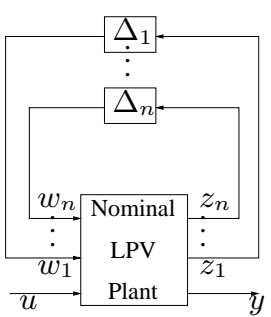

Fig. 1. The uncertain LPV system

which map the plant uncertainty outputs $z_{j}$ into the plant uncertainty inputs $w_{j}$; they are not used as scheduling parameters. Also, the nominal plant is an LPV plant whose coefficients depend on a parameter which can be measured and used for scheduling a controller. We aim to find a gainscheduled controller to ensure the closed loop uncertain LPV system is absolutely stable for all admissible uncertainties.

The methodology of the paper builds on the results of the recent paper [10] which addressed the design of gain-scheduled controllers for uncertain LPV systems with unstructured uncertainty. Unlike the mentioned references using the direct LMI approach, the scheduling algorithm developed in [10] uses the interpolation approach to gainscheduling originated from [11]. This approach has an advantage that the scheduling parameters are not required to be polytopic/polynomial/rational dependent; this allows to avoid the restrictions in [6], [7], [8], [9], e.g., overbounding of the underlying set of system models by a convex set.

In this paper we extend the results of [10] in several directions, the most significant one being the extension to uncertain LPV systems with structured uncertainty. Incorporating the uncertainty structure into the control design algorithm is known to be one way to reduce conservatism of the controller design. It was shown in [12] that a number of robust control design problems for LTI uncertain systems with structured uncertainty can be addressed using the uncertainty description involving time-domain Integral Quadratic Constraints (IQCs). In this paper we extend the method of IQCs to deal with the LPV uncertain system shown in Figure 1. Specifically, our control design uses the fact that the uncertainty inputs under consideration satisfy certain IQCs in which the effect of parameter variations in the system is captured by introducing parameter dependent weightings into the signal norms; thus, the magnitude of the admissible uncertainty is tied to parameter variations. Unlike this paper, in the case of unstructured uncertainty [10] it was possible to use the same IQCs as in the LTI case in the design of 
a gain-scheduled controller. However, it appears that this approach cannot be directly extended to the case of structured uncertainty. The technical contribution of this paper is to develop an approach which overcomes this difficulty.

The method of [10] involves interpolation of controllers designed using parameter-dependent algebraic Riccati equations (AREs). The key idea of the proposed extension to the structured uncertainty case is based on transforming the parametrized AREs into equivalent LMIs. This leads to a scalable controller interpolation algorithm owing to the convexity of these LMIs. Different from the mentioned references on the LMI approach to gain-scheduling, in our case, the LMIs are related to the minimax optimal control design and are convex with respect to inverse Lagrange parameters included to account for the uncertainty constraints. This property serves as the basis for our controller interpolation method. Global convexity with respect to the scheduling variable is not required.

Another important extension of the results of [10] concerns the construction of the control interpolation algorithm. The selection of the Lagrange parameters and corresponding matrix variables is one of the key steps of the controller scheduling scheme proposed in [10]. However, in general [10] gives no indication as to how one can select those unknown design parameters nor it suggests how the operating points for interpolation can be determined. This paper proposes an effective numerical method to solve for those parameters and connects them to the selection of operating points for interpolation. By relaxing the parametrized AREs to inequalities and introducing some additional slack variables, our result reduces the design of a gain-scheduled controller to solving a set of semi-definite programming (SDP) problems involving LMIs.

\section{PROBlEM FORMULATION}

We consider the following class of uncertain LPV systems shown in Figure 1:

$$
\begin{aligned}
& \dot{x}(t)=A(\rho(t)) x(t)+B_{1}(\rho(t)) u(t)+\sum_{j=1}^{n} B_{2 j} w_{j}(t), \\
& z_{j}(t)=C_{j} x(t)+D_{j} u(t), \quad j=1, \cdots, n,
\end{aligned}
$$

where $x(\cdot) \in \mathbb{R}^{n_{x}}$ is the state, $u(\cdot) \in \mathbb{R}^{n_{u}}$ is the control input, $z_{j}(\cdot) \in \mathbb{R}^{q_{j}}$ is the uncertainty output, $w_{j}(\cdot) \in \mathbb{R}^{p_{j}}$ denotes the disturbance, and the time-varying parameter $\rho(\cdot)$ is a continuous function $[0, \infty) \rightarrow \Gamma \triangleq\left[\gamma_{\min }, \gamma_{\max }\right] \subset \mathbb{R}$. The matrices $A(\rho), B_{1}(\rho)$ are continuous on $\Gamma$. To simplify the derivation of the results, we assume $C_{j}^{\prime} D_{j}=0$.

The system (2) is more general than that in [10] in that the control input matrix $B_{1}$ is dependent on the scheduling variable $\rho(\cdot)$, and also the uncertainty is structured, as shown in Figure 1. In accordance with this uncertainty structure, we will assume that $w_{j}(\cdot)$ and $z_{j}(\cdot)$ connected by (1) obey the following 'sector bound' constraints,

$$
\left\|w_{j}(t)\right\| \leq\left\|z_{j}(t)\right\| \quad \forall t \geq 0 ;
$$

$\|\cdot\|$ denotes the standard Euclidean norm. However, in order to make use of the minimax control design method, we need to re-write these constraints in the form of IQCs. First, we select a number of design points $\left\{\gamma_{i}\right\}_{i=1}^{m} \triangleq \Gamma_{d} \subset \Gamma$ and for each $i=1, \cdots, m-1$, consider the functions $\lambda_{i}(t) \triangleq$ $\frac{\gamma_{i+1}-\rho(t)}{\gamma_{i+1}-\gamma_{i}}$ and $\chi_{i}(t)$ such that $\chi_{i}(t)=1$ if $\rho(t) \in\left[\gamma_{i}, \gamma_{i+1}\right)$ and $\chi_{i}(t)=0$ otherwise. In the sequel, we will impose additional conditions on the design points. For now, we introduce the following notion of admissible uncertainty for the system (2).

Definition 1: Let $d_{\nu, j}(i)>0, \nu, j=1, \cdots, n, i=$ $1, \cdots, m-1$, be given constants. An uncertainty input $w(\cdot)=\left[w_{1}^{\prime}(\cdot), \cdots, w_{n}^{\prime}(\cdot)\right]^{\prime}$ of the form (1) is an admissible input for the LPV system (2) if the following condition holds. For any locally square integrable control input $u(\cdot)$ and a corresponding solution to (2) on $[0,+\infty)$, there exists a sequence $\left\{t_{l}\right\}_{l=1}^{\infty}$ such that $t_{l} \rightarrow+\infty, t_{l} \geq 0$ and

$$
\begin{aligned}
& \int_{0}^{t_{l}}\left[\begin{array}{rr}
\lambda_{i}^{\nu-1}(t)\left[1-\lambda_{i}(t)\right]^{n-\nu} \chi_{i}(t) \\
& \times\left(\left\|w_{j}(t)\right\|^{2}-\left\|z_{j}(t)\right\|^{2}\right)
\end{array}\right] d t \leq d_{\nu, j}(i), \\
& \nu, j=1, \cdots, n, \quad i=1, \cdots, m-1 \text {. }
\end{aligned}
$$

Note that $t_{l}$ may be equal to infinity. The set of all admissible uncertainty inputs $w(\cdot)$ will be denoted $\mathcal{W}_{L P V}$.

Conditions (4) are generalized time domain IQCs. Originally introduced by Yakubovich, the IQC uncertainty description has proved useful in a number of controller synthesis problems for LTI systems [12]. In particular, it has enabled the development of the minimax control approach to the design of robust controllers for uncertain LTI systems. In this paper we will employ these results on the minimax LTI control in the derivation of guaranteed cost control results for the more general uncertain LPV system (2).

Compared to the standard IQCs [12], the constraints (4) involve the weightings $\lambda_{i}^{\nu-1}(t)\left[1-\lambda_{i}(t)\right]^{n-\nu} \chi_{i}(t)$. Owing to the indicator function $\chi_{i}(t)$ of the interval $\left[\gamma_{i}, \gamma_{i+1}\right)$ the constraints (4) restrict attention to the mismatch in the energy of uncertainty inputs and uncertainty outputs in this particular part of the system envelop. Also, the weighting $\lambda_{i}^{\nu-1}(t)[1-$ $\left.\lambda_{i}(t)\right]^{n-\nu}$ helps to relate the allowable mismatch between the uncertainty inputs and uncertainty outputs to the variations of the scheduling variable $\rho(\cdot)$ in the interval $\left[\gamma_{i}, \gamma_{i+1}\right)$.

Note some special cases of the IQCs (4). As the first special case, we note that the pairs of uncertainty inputs and uncertainty outputs satisfying (3), also satisfy (4), and hence are admissible in the sense of Definition 1. For instance, the norm-bounded time-varying uncertainty satisfies the IQCs (4) trivially. The second special case is where $\rho(t) \equiv \gamma=$ const $\in\left[\gamma_{i^{*}}, \gamma_{i^{*}+1}\right)$. In this case, $\chi_{i}(t) \equiv 0$ for all $i \neq i^{*}$, $\lambda_{i^{*}}(t) \equiv\left(\gamma_{i^{*}+1}-\gamma\right) /\left(\gamma_{i^{*}+1}-\gamma_{i^{*}}\right)=$ const, and the IQCs (4) reduce to (assuming $\lambda_{i^{*}}^{\nu-1}\left(1-\lambda_{i^{*}}\right)^{n-\nu} \neq 0$ ): for $\nu, j=1, \ldots, n$,

$$
\int_{0}^{t_{l}}\left(\left\|w_{j}(t)\right\|^{2}-\left\|z_{j}(t)\right\|^{2}\right) d t \leq \frac{d_{\nu, j}\left(i^{*}\right)}{\lambda_{i^{*}}^{\nu-1}\left(1-\lambda_{i^{*}}\right)^{n-\nu}} .
$$

That is, the condition (5) is a standard IQC

$$
\int_{0}^{t_{l}}\left\|w_{j}(t)\right\|^{2} d t \leq \int_{0}^{t_{l}}\left\|z_{j}(t)\right\|^{2} d t+d_{j}, j=1, \cdots, n,
$$

in which $d_{j}$ 's are some suitable constants. 
Associated with the LPV system (2) and the uncertainty class $\mathcal{W}_{L P V}$, consider the following set of state-feedback gain-scheduled controllers $u=u(t, \rho(t), x)$ of the form

$$
\begin{aligned}
\dot{x}_{c}(t) & =A_{c}(\rho(t)) x_{c}(t)+B_{c}(\rho(t)) x(t), \\
u(t) & =K_{c}(\rho(t)) x_{c}(t),
\end{aligned}
$$

where $x_{c} \in \mathbf{R}^{n_{c}}$ is the controller state vector. We aim to design a state-feedback controller of the form (7) for the uncertain LPV system (2) such that under the condition $\rho(t) \in \Gamma \forall t \in[0, \infty)$, the closed-loop uncertain system is absolute stable for all admissible disturbances $w(\cdot) \in \mathcal{W}_{L P V}$ in the sense of Definition 2 given below. The design should utilize only a finite number of design points $\Gamma_{d} \subset \Gamma$.

Definition 2: The system (2), (4) is said to be absolutely stabilizable via state feedback control if there exists a controller (7) and a constant $c>0$ such that for any initial conditions $\left[x^{\prime}(0), x_{c}^{\prime}(0)\right]^{\prime}$ and any admissible uncertainty inputs $w_{j}(\cdot)$, the signals $x(\cdot), x_{c}(\cdot), w_{j}(\cdot)$ are uniformly bounded,

$$
\begin{aligned}
\|x(\cdot)\|_{2}^{2} & +\left\|x_{c}(\cdot)\right\|_{2}^{2}+\sum_{i=1}^{n}\left\|w_{j}(\cdot)\right\|_{2}^{2} \\
& \leq c\left(\|x(0)\|^{2}+\left\|x_{c}(0)\right\|^{2}\right) .
\end{aligned}
$$

Here we used the notation $\|q(\cdot)\|_{2}^{2} \triangleq \int_{0}^{\infty}\|q(t)\|^{2} d t$.

The IQCs (6) were considered in the minimax optimal control approach to the design of robust controllers for the LTI uncertain systems [13], [12], and in the case of $\rho(t)=$ const, the stability property stated in Definition 2 reduces to the absolute stability property for LTI uncertain systems considered in those references. Hence, if it were possible to 'freeze' the scheduling parameter $\rho$, the controller could be constructed using the minimax control design method. It is important to note that the condition (6) is independent of $\rho$. This observation is crucial for the controller design that follows. It will allow us to proceed with the controller design as if the parameters of the system were fixed, and then derive additional conditions on the rate of parameter variation of the system (2) under which the underlying uncertain LPV system (2), (4) governed by this controller is absolutely stable.

\section{REVIEW OF THE MINIMAX CONTROL DESIGN}

\section{A. Minimax control design for a fixed parameter system}

Let us fix $\gamma \in \Gamma$ and assume that $\left(A(\gamma), B_{1}(\gamma)\right)$ is stabilizable. Consider the fixed parameter uncertain linear system with structured uncertainty

$$
\begin{aligned}
& \dot{x}=A(\gamma) x+B_{1}(\gamma) u+\sum_{j=1}^{n} B_{2 j} w_{j}, \\
& z_{j}=C_{j} x+D_{j} u, \quad j=1, \cdots, n .
\end{aligned}
$$

As observed above, the IQCs (4) in this case reduce to the IQCs (5), or equivalently the IQCs (6); we denote the set of uncertainties of the form (1) for the system (9) satisfying (6) by $\mathcal{W}_{\gamma}$. This allows us to apply the minimax optimal control design technique of [13], [12] to the system (9) subject to the IQCs (6). Consider the following quadratic cost:

$$
J(u, w):=\int_{0}^{\infty}\left(x^{\prime} M x+u^{\prime} N u\right) d t,
$$

where $M>0$ and $N>0$ are given weighting matrices. The minimax optimal state-feedback control law $u^{*}$ minimizes the worst case of the performance cost (10) for this system,

$$
\begin{aligned}
u^{*} & :=\arg \inf _{u} \mu(u), \\
\mu(u) & :=\sup _{w \in \mathcal{W}_{\gamma}}\{J(u, w): \text { subject to (9) }\} .
\end{aligned}
$$

Since for now we assume that $\gamma$ is fixed, the results of [13] can be applied to obtain a solution to the problem (11).

Consider the matrix Riccati equation:

$$
\begin{gathered}
A^{\prime}(\gamma) X+X A(\gamma)-X\left[B_{1}(\gamma)\left(N+\sum_{j=1}^{n} \tau_{j} D_{j}^{\prime} D_{j}\right)^{-1} B_{1}^{\prime}(\gamma)\right. \\
\left.-\sum_{j=1}^{n} \tau_{j}^{-1} B_{2 j} B_{2 j}^{\prime}\right] X+M+\sum_{j=1}^{n} \tau_{j} C_{j}^{\prime} C_{j}=0,
\end{gathered}
$$

where $\tau_{j}>0$, and define the set $\Pi(\gamma)$ consisting of all $\tau=\left(\tau_{1}, \cdots, \tau_{n}\right), \tau_{j}>0$, for which (12) admits a stabilizing solution $X(\tau)=X^{\prime}(\tau)>0$.

Lemma 1 ([13], [12]): For a given $\gamma \in \Gamma$, if $\Pi(\gamma) \neq \emptyset$, then the solution to the problem (11) is given by

$$
\begin{aligned}
& u^{*}(x)=-\left(N+\sum_{j=1}^{n} \tau_{j}^{*} D_{j}^{\prime} D_{j}\right)^{-1} B_{1}^{\prime}(\gamma) X\left(\tau^{*}\right) x, \\
& \tau^{*}=\left(\tau_{1}^{*}, \cdots, \tau_{n}^{*}\right):=\arg \inf _{\tau \in \Pi(\gamma)} x_{0}^{\prime} X(\tau) x_{0} .
\end{aligned}
$$

The worst-case cost defined in (11), is given by

$$
\mu\left(u^{*}\right)=x_{0}^{\prime} X\left(\tau^{*}\right) x_{0}+\sum_{j=1}^{n} \tau_{j}^{*} d_{j} .
$$

Conversely, if $\Pi(\gamma)=\emptyset$, then the optimal minimax controller $u^{*}$ does not exist and $\mu(u)=\infty$ for all statefeedback controllers $u(\cdot)$.

\section{B. Minimax control design for an LPV system: small param-} eter variations

The minimax optimal controller (13) for a fixed $\gamma \in \Gamma$ can be used in the derivation of a controller for the LPV system (2), provided parameter variations are sufficiently small, i.e., $\rho(t) \approx \gamma \forall t \geq 0$. In this case small parametric perturbations of the matrices $A(\cdot), B_{1}(\cdot)$ can be treated as disturbances, and the LPV system (2) can be regarded as a perturbation of a linear fixed-parameter system. Let us fix $\gamma \in \Gamma$, and rewrite the uncertain LPV system (2) as follows

$$
\dot{x}=A(\gamma) x+B_{1}(\gamma) u+\sum_{j=1}^{n} B_{2 j} w_{j}+\beta_{1} \xi_{1}+\beta_{2} \xi_{2},
$$

where $\beta_{1}>0, \beta_{2}>0$ are some constants, $\xi_{1}(t):=$ $\left(1 / \beta_{1}\right)[A(\rho(t))-A(\gamma)] x(t)$, and $\xi_{2}(t):=\left(1 / \beta_{2}\right)\left[B_{1}(\rho(t))-\right.$ $\left.B_{1}(\gamma)\right] u(t)$. The minimax optimal controller can now be designed based on the representation (16); it will guarantee robustness of the system (16) against both the disturbance $w_{j}(\cdot)$ and the parameter variation in $A(\cdot), B_{1}(\cdot)$. To proceed, the set $\mathcal{W}$ of uncertainties (1) for the system (16) satisfying the uncertainty constraint (6) needs to be augmented in order to account for the structured uncertainty $w_{j}(\cdot), \xi_{1}(\cdot) \xi_{2}(\cdot)$. Associated with the modified state equation (16), we consider the following IQCs, additional to the IQCs (6): 


$$
\int_{0}^{t_{l}}\left\|\xi_{1}\right\|^{2} d t \leq \int_{0}^{t_{l}}\|x\|^{2} d t, \quad \int_{0}^{t_{l}}\left\|\xi_{2}\right\|^{2} d t \leq \int_{0}^{t_{l}}\|u\|^{2} d t .
$$

Here $\left\{t_{l}\right\}$ is the sequence from Definition 1. In (17), $x(\cdot)$ is the state process generated by the fixed parameter LPV system (2) (or equivalently (16)) driven by $w_{j}(\cdot), j=1, \cdots, n$, and $u(\cdot)$. Note that when $\rho(\cdot) \equiv \gamma=$ const, then $\xi_{1}(\cdot) \equiv$ $0, \xi_{2}(\cdot) \equiv 0$ and the structured uncertainty constraints (17) are trivially satisfied, hence the system uncertainty is subject to the IQCs (6) only. Also, in contrast to [10], to reduce conservatism, the uncertainty due to deviations of the system matrices from the fixed values is accounted for using separate constraints from those imposed on the disturbances $w_{j}$.

The introduced modification allows us to apply the minimax optimal state-feedback control design technique to the LPV system (16). The resulting controller, due to its robustness properties, will stabilize the original uncertain LPV system provided the parameter $\rho(\cdot)$ varies in a small neighborhood of $\gamma$. The size of the neighborhood depends on the choice of $\beta_{1}, \beta_{2}$ and also on the parameter $\gamma \in \Gamma$. We conclude this discussion by formally stating the result.

Theorem 1: Let $\beta_{1}, \beta_{2}$ and $\gamma \in \Gamma$ be chosen so that

$$
\begin{aligned}
\|A(\rho(t))-A(\gamma)\| & <\beta_{1}, \quad \forall t \in[0, \infty), \\
\left\|B_{1}(\rho(t))-B_{1}(\gamma)\right\| & <\beta_{2}, \quad \forall t \in[0, \infty) ;
\end{aligned}
$$

$\|\cdot\|$ denotes the largest singular value. Consider the ARE

$$
\begin{aligned}
& A^{\prime}(\gamma) X+X A(\gamma)+M+\left(\sum_{j=1}^{n} \tau_{j} C_{j}^{\prime} C_{j}+\tau_{n+1} I\right) \\
& -X\left[B_{1}(\gamma)\left(N+\sum_{j=1}^{n} \tau_{j} D_{j}^{\prime} D_{j}+\tau_{n+2} I\right)^{-1} B_{1}^{\prime}(\gamma)\right. \\
& \left.-\left(\sum_{j=1}^{n} \tau_{j}^{-1} B_{2 j} B_{2 j}^{\prime}+\tau_{n+1}^{-1} \beta_{1}^{2} I+\tau_{n+2}^{-1} \beta_{2}^{2} I\right)\right] X=0,
\end{aligned}
$$

and suppose that the correspondingly modified set $\Pi(\gamma)=$ $\left\{\vec{\tau}=\left(\tau_{1}, \cdots, \tau_{n+2}\right): \tau_{j}>0,(20)\right.$ admits a stabilizing solution $\left.X=X^{\prime}>0\right\}$ is not empty. Then the minimax optimal controller

$$
\begin{aligned}
& u_{\gamma}^{*}=-\left(N+\sum_{j=1}^{n} \tau_{j}^{*} D_{j}^{\prime} D_{j}+\tau_{n+2}^{*} I\right)^{-1} B_{1}^{\prime}(\gamma) X\left(\vec{\tau}^{*}\right) x,(21) \\
& \vec{\tau}^{*}=\left(\tau_{1}^{*}, \ldots, \tau_{n+2}^{*}\right):=\arg \inf _{\vec{\tau} \in \Pi(\gamma)}\left[x_{0}^{\prime} X(\vec{\tau}) x_{0}+\sum_{j=1}^{n} \tau_{j} d_{j}\right],
\end{aligned}
$$

absolutely stabilizes the uncertain LPV system (2), (6) and yields the robust performance bound

$$
\mu:=\sup _{w \in \mathcal{W}} J\left(u_{\gamma}^{*}, w\right) \leq x_{0}^{\prime} X\left(\vec{\tau}^{*}\right) x_{0}+\sum_{j=1}^{n} \tau_{j}^{*} d_{j} .
$$

\section{Gain-scheduling of Minimax Optimal CONTROLLERS}

In this section we use the results of the previous section in order to introduce a controller schedule for the uncertain LPV system (2), (4). We will assume that the selected set of design points $\Gamma_{d} \subset \Gamma$ satisfies the following conditions:
1) Each pair $\left(A\left(\gamma_{i}\right), B_{1}\left(\gamma_{i}\right)\right)$ is stabilizable.

2) For each $t \in[0, \infty)$, there exists at least one $i \in$ $\{1, \ldots, m\}$ and $\beta_{1, i}>0, \beta_{2, i}>0$ such that the inequalities (18), (19) hold with $\beta_{1}=\beta_{1, i}, \beta_{2}=\beta_{2, i}$.

3) $\forall i=1, \ldots, m$, the ARE (20) admits a stabilizing solution $X_{i}>0$ for some $\tau_{1, i}>0, \cdots, \tau_{n+2, i}>0$ and selected $\beta_{1, i}>0, \beta_{2, i}>0$; i.e, the corresponding sets $\Pi\left(\gamma_{i}\right)$ must be not empty for the selected $\beta_{1, i}, \beta_{2, i}$.

In section $\mathrm{V}$, we will show that the last assumption can be reformulated as a certain set of SDP problems. This will allow for this assumption to be verified numerically.

The above assumptions allow us to use Theorem 1 to compute $m$ minimax optimal controllers of the form (21) for the uncertain LPV plants (16) subject to (6), (17) for each $\gamma_{i}$. Let $u_{i}^{*}$ and $\mu_{i}$ denote such minimax optimal controller (21) and the corresponding worst-case cost in (22). Also, let $\vec{\tau}_{i}=\left(\tau_{1}(i), \cdots, \tau_{n+2}(i)\right)$ and $X_{i}$ denote the value $\vec{\tau}^{*}$ in (22) and the corresponding matrix $X\left(\vec{\tau}^{*}\right)$, respectively. Due to robustness of each such controller, we can ascertain the existence of a neighborhood $U_{i} \subset \mathbb{R}$ of each design point $\gamma_{i} \in \Gamma_{d}$, such that all closed loop properties in Theorem 1 hold under the condition $\{\rho(t) ; 0 \leq t<\infty\} \subset U_{i}$. Then, choosing the design points $\left\{\gamma_{i}\right\}$ such that $\Gamma \subset \cup_{i=1}^{m} U_{i}$, allows us to conclude that any fixed parameter system in which $\rho(t)=\gamma \in \Gamma$ is stabilizable by one of the designed controllers; this property corresponds to the stability covering condition [11]. The proposed controller schedule is

$$
u_{n s}(t, \gamma, x):=u_{\nu}^{*}(x)
$$

$\nu:=\arg \min _{i}\left\{\mu_{i}:\left\|A(\gamma)-A\left(\gamma_{i}\right)\right\|<\beta_{1, i}, \| B_{1}(\gamma)-\right.$ $\left.B_{1}\left(\gamma_{i}\right) \|<\beta_{2, i}\right\}$; that is, if $\gamma \in \cap_{i} U_{i}$, then we choose a controller which guarantees a better performance.

However, if the controller (23) is applied to the LPV system (2), its gain may become discontinuous at the time instant when the trajectory of $\rho(t)$ exits the set $U_{i}$ and enters the set $U_{i+1}$. In a practical situation, switching control gains may lead to undesirable transients. We therefore propose a continuous interpolation of the minimax controllers $\left\{u_{i}^{*}\right\}_{i=1}^{m}$, following the reference [11]. Our interpolation technique follows [10] and differs from that used in [11] in that it preserves, along with stability of the nominal closed loop system, the property of interpolants to guarantee a bound on the worst-case quadratic performance cost and hence robustness. Although the idea behind this particular robustness preserving controller interpolation was proposed in [10], this paper revisits the design of [10] from a different technical perspective. This allows us to remove some limitations of the approach of [10], the main limitation being the lack of tools to address the structure of uncertain perturbations.

\section{A. The design of a gain-scheduled controller}

We first introduce an interpolated controller for the fixed parameter system (9), and then show that the proposed controller can be applied to the LPV system (2). Consider the uncertain fixed parameter system (9), and assume $\gamma \in$ 
$U_{i} \cap U_{i+1}$, that is

$$
\begin{aligned}
\left\|A(\gamma)-A\left(\gamma_{k}\right)\right\|<\beta_{1, k} & \text { for } \quad k=i, i+1, \\
\left\|B_{1}(\gamma)-B_{1}\left(\gamma_{k}\right)\right\|<\beta_{2, k} & \text { for } \quad k=i, i+1 .
\end{aligned}
$$

Lemma 2: Suppose $\left(\gamma_{i}, \vec{\tau}_{i}, X_{i}\right)$ and $\left(\gamma_{i+1}, \vec{\tau}_{i+1}, X_{i+1}\right)$ satisfy (20), where $\vec{\tau}_{k}=\left(\tau_{1}(k), \cdots, \tau_{n+2}(k)\right), k=i, i+1$. Also, suppose $A(\gamma), B_{1}(\gamma)$ satisfy (24), (25). Define for $\lambda \in[0,1]$

$$
\begin{gathered}
N_{\lambda}=N+\sum_{j=1}^{n} \tau_{\lambda, j} D_{j}^{\prime} D_{j}, \quad X_{\lambda}=\left[\lambda X_{i}^{-1}+(1-\lambda) X_{i+1}^{-1}\right]^{-1} \\
\tau_{\lambda, j}=\left[\lambda \tau_{j}^{-1}(i)+(1-\lambda) \tau_{j}^{-1}(i+1)\right]^{-1}
\end{gathered}
$$

Then $\left(\tau_{\lambda}, X_{\lambda}\right), \tau_{\lambda} \triangleq\left(\tau_{\lambda, 1}, \cdots, \tau_{\lambda, n}\right)$, satisfies

$$
\begin{gathered}
A^{\prime}(\gamma) X_{\lambda}+X_{\lambda} A(\gamma)+\sum_{j=1}^{n} \tau_{\lambda, j}^{-1} X_{\lambda} B_{2 j} B_{2 j}^{\prime} X_{\lambda} \\
-X_{\lambda} B_{1}(\gamma) N_{\lambda}^{-1} B_{1}^{\prime}(\gamma) X_{\lambda}+M+\sum_{j=1}^{n} \tau_{\lambda, j} C_{j}^{\prime} C_{j}<0
\end{gathered}
$$

Definition 3: Assuming (24-25), define the interpolated controller by letting $\lambda=\left(\gamma_{i+1}-\gamma\right) /\left(\gamma_{i+1}-\gamma_{i}\right)$ and

$$
u_{\lambda}:=-N_{\lambda}^{-1} B_{1}^{\prime}(\gamma) X_{\lambda} x
$$

The minimax optimality properties of the interpolants do not imply that the interpolated controller delivers a bounded worst-case performance. The robust controller interpolation problem is no different in this regard from the stability interpolation problem discussed in [11]. However, the robust stability and performance of the uncertain fixed parameter system (9), governed by the controller (28), can be evaluated following the idea of [10]. By proving that the worstcase quadratic performance of the fixed parameter uncertain system is bounded, we can ascertain stability of the closed loop system.

Theorem 2: Under conditions (24) and (25), the interpolated controller $u_{\lambda}(\cdot)$ given in (28) robustly stabilizes the uncertain fixed parameter system (9). The corresponding worst-case cost is bounded as follows,

$$
\bar{\mu}(\gamma):=\sup _{w \in \mathcal{W}} J\left(u_{\lambda}, w\right) \leq \lambda \mu_{i}+(1-\lambda) \mu_{i+1} .
$$

\section{B. Stabilizing properties of the interpolated controller}

To study stabilizing properties of the interpolated controller in relation to the underlying LPV system (2), for simplicity, we will assume that $\left[\gamma_{i}, \gamma_{i+1}\right] \in U_{i} \cap U_{i+1}$ for all $i=1, \ldots, m-1$. This technical assumption can be easily relaxed at the expense of considering a somewhat more complex piecewise continuous interpolation rule as in [10]. Under this simplifying assumption, the interpolation rule simplifies to the following LPV controller gain schedule:

$$
\begin{gathered}
u^{s}(t, \rho(t), x)=-\left(N^{s}\right)^{-1} B_{1}^{\prime}(\rho(t)) X^{s} x \\
\text { where } \quad N^{s}=N+\sum_{j=1}^{n} \tau_{j}^{s} D_{j}^{\prime} D_{j}, \quad \rho(t) \in\left[\gamma_{i}, \gamma_{i+1}\right), \\
\left(\tau_{j}^{s}, \quad X^{s}\right):=\left(\tau_{\lambda(t)}, X_{\lambda(t)}\right), \quad \lambda(t)=\frac{\gamma_{i+1}-\rho(t)}{\gamma_{i+1}-\gamma_{i}}
\end{gathered}
$$

It follows from Theorem 2 that the controller (30) stabilizes the uncertain system (9) with constant but a priori unknown parameter $\gamma \in \Gamma$. We now show that this gainscheduled controller robustly stabilizes the original uncertain LPV system (2), (4), provided the scheduling variable $\rho(t)$ varies sufficiently slowly.

$$
\begin{aligned}
& \text { Let } \tilde{\tau}^{s}=\prod_{v=1}^{n} \tau_{v}^{s} \text {. Then we can write } \tau_{j}^{s} / \tilde{\tau}^{s} \text { as } \\
& \frac{\tau_{j}^{s}}{\tilde{\tau}^{s}}=\sum_{i=1}^{m-1} \sum_{v=1}^{n} \lambda^{v-1}(t)[1-\lambda(t)]^{n-v} \theta_{v, j}(i) \chi_{i}(t) .
\end{aligned}
$$

where $\theta_{v, j}(i)>0$ is a constant defined by $\tau_{l}^{-1}(i), \tau_{l}^{-1}(i+$ 1), $l \neq j$, when $\gamma \in\left[\gamma_{i}, \gamma_{i+1}\right)$.

Lemma 3: Under the IQC conditions (4), the follwing IQCs also hold for $j=1, \cdots, n$ :

$$
\int_{0}^{t_{l}} \frac{\tau_{j}^{s}}{\tilde{\tau}^{s}}\left(\left\|w_{j}(t)\right\|^{2}-\left\|z_{j}(t)\right\|^{2}\right) d t \leq \sum_{i=1}^{m-1} \sum_{v=1}^{n} \theta_{v, j}(i) d_{v, j}(i),
$$

From Lemma 3, the main result of the paper follows.

Theorem 3: Suppose that the time-varying parameter $\rho(\cdot)$ of the uncertain LPV system (2) satisfies the condition

$$
\sup _{t}|\dot{\rho}(t)|<\frac{q \eta}{\delta}, \quad \delta:=\sup _{\gamma \in \Gamma \backslash \Gamma_{d}}\left\|\frac{d}{d \gamma}\left(X^{s} / \tilde{\tau}^{s}\right)\right\|,
$$

where $q \in[0,1)$ is a constant, and $\eta>0$ is defined as

$$
\eta:=\min _{\gamma \in \Gamma} \varrho\left\{\frac{1}{\tilde{\tau}^{s}}\left[M+X^{s} B_{1}(\gamma)\left(N^{s}\right)^{-1} N\left(N^{s}\right)^{-1} B_{1}^{\prime}(\gamma) X^{s}\right]\right\} ;
$$

$\varrho(\cdot)$ denotes the smallest eigenvalue of a matrix. Then, the closed loop uncertain LPV system consisting of the uncertain LPV system (2), (4) and the gain-scheduled controller (30) is absolutely stable for all disturbances $w(\cdot)$ satisfying the IQCs (4). Furthermore, the following worst-case performance bound can be ascertained

$$
\begin{aligned}
\sup _{w \in \mathcal{W}_{L P V}} J\left(u^{s}, w\right) & \leq \frac{\tilde{\tau}_{\max }}{1-q}\left[\frac{1}{\tilde{\tau}^{s}\left(\gamma_{\circ}\right)} x^{\prime}(0) X^{s}\left(\gamma_{\circ}\right) x(0)\right. \\
& \left.+\sum_{j=1}^{n} \sum_{i=1}^{m-1} \sum_{v=1}^{n} \theta_{v, j}(i) d_{v, j}(i)\right]
\end{aligned}
$$

where $\gamma_{\circ}:=\rho(0), \tilde{\tau}_{\max }:=\max _{\gamma \in \Gamma} \tilde{\tau}^{s}$.

As observed in Section II, the uncertain perturbations of the form (1) satisfying the constraint (3) also belong to the set $\mathcal{W}_{L P V}$. The definition of $\mathcal{W}_{L P V}$ involves the parameters $d_{\nu, j}(i)$, however embedding the constraints (3) into (4) can be achieved by selecting arbitrarily small $d_{\nu, j}(i)$. Therefore the conditions of Theorem 3 imply robust stabilization of the underlying system (2) subject to the structured uncertainty (1), (3) by means of the gain-scheduled controller (30).

The bound (34) depends on the design parameters $\beta_{1, i}, \beta_{2, i}$. Since $A(\gamma), B_{1}(\gamma)$ are continuous, conditions (24), (25) implicitly relate the parameters $\beta_{1, i}, \beta_{2, i}$ to the size of cover sets $U_{i}$. In Section $\mathrm{V}$, we will introduce a set of SDP problems from which $\beta_{1, i}, \beta_{2, i}$ and the corresponding scaling parameters can be found numerically. 
Finally, note that for the fixed parameter system (9), we have $\tilde{\tau}_{\max }=\tilde{\tau}^{s}$ and $\dot{\rho}=0$. Therefore $q=0$ and the IQCs (4) are equivalent to IQCs (6) since $\lambda$ is a constant, and the cost bound (35) reduces to the bound (29) in Theorem 2.

\section{LMI REALIZATION}

As shown in Sections III, IV, the proposed gain-scheduling controller design involves solving a set of parameterized game-type AREs for $i=1, \cdots, n$,

$$
\begin{aligned}
& A^{\prime}\left(\gamma_{i}\right) X_{i}+X_{i} A\left(\gamma_{i}\right)-X_{i}\left[B_{1}\left(\gamma_{i}\right) N_{\alpha i}^{-1} B_{1}^{\prime}\left(\gamma_{i}\right)\right. \\
& \left.-\left(\sum_{j=1}^{n} \tau_{j}^{-1}(i) B_{2 j} B_{2 j}^{\prime}+\tau_{n+1}^{-1}(i) \beta_{1, i}^{2} I+\tau_{n+2}^{-1}(i) \beta_{2, i}^{2} I\right)\right] X_{i} \\
& \quad+M+\sum_{j=1}^{n} \tau_{j}(i) C_{j}^{\prime} C_{j}+\tau_{n+1}(i) I=0,
\end{aligned}
$$

where $N_{\alpha k}=N+\sum_{j=1}^{n} \tau_{j}(k) D_{j}^{\prime} D_{j}+\tau_{n+2}(k) I$.

Define the following variables:

$$
\begin{aligned}
& \bar{X}_{i}=X_{i}^{-1}, \quad \bar{\tau}_{j}(i)=\tau_{j}^{-1}(i), \quad j=1, \cdots, n+2, \\
& \hat{\tau}_{1}(i)=\tau_{n+1}^{-1}(i) \beta_{1, i}^{2}, \quad \hat{\tau}_{2}(i)=\tau_{n+2}^{-1}(i) \beta_{2, i}^{2} .
\end{aligned}
$$

By applying the technique used in [14], under the assumption that the pair $\left(A\left(\gamma_{i}\right), B_{1}\left(\gamma_{i}\right)\right)$ is stabilizable for $i=$ $1, \cdots, m$, solving the ARE (36) is equivalent to solving the following SDP problems in the variables $W_{i}, \bar{X}_{i}, \bar{F}_{i}$, $\bar{\tau}_{1}(i), \cdots, \bar{\tau}_{n+2}(i), \hat{\tau}_{1}(i), \hat{\tau}_{2}(i)$ :

$$
\begin{aligned}
& \min \operatorname{Trace}\left(W_{i}\right) \\
& {\left[\begin{array}{cc}
\mathbf{M}_{11} & \mathbf{M}_{12} \\
\star & \mathbf{M}_{22}
\end{array}\right]<0, \quad\left[\begin{array}{cc}
W_{i} & I_{n_{x}} \\
\star & \bar{X}_{i}
\end{array}\right]>0,}
\end{aligned}
$$

where $\quad \mathbf{M}_{11}=\bar{X}_{i} A^{\prime}\left(\gamma_{i}\right)+A\left(\gamma_{i}\right) \bar{X}_{i}+\sum_{j=1}^{n} \bar{\tau}_{j}(i) B_{2 j} B_{2 j}^{\prime}$

$$
\begin{array}{r}
+\hat{\tau}_{1}(i) I+\hat{\tau}_{2}(i) I+B_{1}\left(\gamma_{i}\right) \bar{F}_{i}+\bar{F}_{i}^{\prime} B_{1}^{\prime}\left(\gamma_{i}\right), \\
\mathbf{M}_{12}=\left[\bar{X}_{i} M^{1 / 2}, \bar{F}_{i}^{\prime} N^{1 / 2}, \bar{X}_{i} C_{1}^{\prime}+\bar{F}_{i}^{\prime} D_{1}^{\prime}, \cdots,\right. \\
\left.\bar{X}_{i} C_{n}^{\prime}+\bar{F}_{i}^{\prime} D_{n}^{\prime}, \bar{X}_{i}, \bar{F}_{i}^{\prime}\right], \\
\mathbf{M}_{22}=-\operatorname{diag}\left[I_{n_{x}}, I_{n_{u}}, \bar{\tau}_{1}(i) I_{q_{1}}, \cdots, \bar{\tau}_{n}(i) I_{q_{n}},\right. \\
\left.\bar{\tau}_{n+1}(i) I_{n_{x}}, \bar{\tau}_{n+2}(i) I_{n_{u}}\right] .
\end{array}
$$

This equivalence can be proved in a similar fashion to [14].

Solving the above SDP problems allows to obtain $X_{i}$, $\tau_{1}(i), \cdots, \tau_{n}(i), \beta_{1, i}, \beta_{2, i}$ from (37) systematically. Then the state feedback gain $K$ is readily scheduled from (30).

The controller interpolation algorithm proposed in [10] was essentially a two-step algorithm. First the parameters $\beta_{1, i}, \beta_{2, i}$ were to be selected, then the parametrized AREs (36) corresponding to these parameters were to be solved, while no indication was given as to how one should choose $\beta_{1, i}, \beta_{2, i}$. In contrast, the algorithm proposed above provides a convex approach to solving for $\beta_{1, i}, \beta_{2, i}$ and the corresponding scaling parameters. This makes this algorithm much easier to implement in practical systems.

Finally note that the controller interpolation schedule (28) can be generalized to uncertain LPV systems whose dynamics are governed by varying vector parameters. The details of this extension will be reported elsewhere. We only note that the proposed improved scheme employs a substantially reduced number of interpolants, compared to [10].

\section{CONCLUSIONS}

In this paper we have considered a continuous gainscheduling problem for uncertain LPV systems. Our result extends the robust gain-scheduling via interpolation approach developed in [10] to the case of uncertain systems in which the uncertainty is structured. The key idea behind the proposed controller interpolation algorithm is to transform the parameterized algebraic Riccati inequalities into equivalent LMIs, so that the natural convexity properties of the LMI feasibility sets can be exploited. It has been shown that the proposed controller robustly stabilizes the fixedparameter uncertain LPV system, yielding a bounded worstcase quadratic cost, and as a result, the uncertain closed loop LPV system governed by the interpolated controller is absolutely stable provided the system parameters vary sufficiently slowly. A bound on the rate of scheduling parameter variations has been obtained. Furthermore, we provide a systematic way to solve the underlying parameterized AREs, which transforms the proposed gain-scheduled controller design to solving a set of SDP problems involving LMIs.

\section{REFERENCES}

[1] W. J. Rugh, "Analytical framework for gain scheduling," IEEE Contr. Syst. Mag., vol. 11, no. 1, pp. 79-84, 1991.

[2] J. Shamma and M. Athans, "Gain scheduling: Potential hazards and possible remedies," IEEE Control System Magazine, vol. 12, no. 3, pp. 101-107, 1992.

[3] P. Apkarian and H. D. Tuan, "Parameterized LMIs in control theory," SIAM J. Contr. Optim., vol. 38, no. 4, pp. 1241-1264, 2000.

[4] C. W. Scherer, "Relaxations for robust linear matrix inequality problems with verifications for exactness," SIAM Journal on Matrix Analysis and Applications, vol. 27, no. 2, pp. 365-395, 2005.

[5] P. Apkarian and P. Gahinet, "A convex characterization of gainscheduled $H_{\infty}$ controllers," IEEE Trans. Automat. Contr., vol. 40, pp. 853-864, 1995.

[6] P. Pellanda, P. Apkarian, and H. D. Tuan, "Missile autopilot design via a multi-channel LFT/LPV control method," Int. J. Robust Nonlinear Control, vol. 12, pp. 1-20, 2002.

[7] P. A. Bliman, "A convex approach to robust stability for linear systems with uncertain scalar parameters," SIAM J. Contr. Optim., vol. 42, no. 6, pp. 2016-2042, 2004.

[8] M. Dinh, G. Scorletti, V. Fromion, and E. Magarotto, "Parameter dependent $H_{\infty}$ control by finite dimensional LMI optimization: application to trade-off dependent control," Int. J. Robust Nonlinear Contr. vol. 15, no. 9, pp. 383-406, 2005.

[9] P. Apkarian, P. Gahinet, and G. Becker, "Self-scheduled $H_{\infty}$ control of linear parameter-varying systems: A design example," Automatica, vol. 31, no. 9, pp. 1251-1261, 1995.

[10] M. Yoon, V. Ugrinovskii, and M. Pszczel, "Gain-scheduling of minimax optimal state-feedback controllers for uncertain linear parametervarying systems," IEEE Transactions Autom. Contr., vol. 52, no. 2, pp. 311-317, 2007.

[11] D. J. Stilwell and W. J. Rugh, "Interpolation of observer state feedback controllers for gain scheduling," IEEE Trans. Automat. Contr., vol. 44 no. 6, pp. 1225-1229, 1999.

[12] I. R. Petersen, V. A. Ugrinovskii, and A. V. Savkin, Robust Control Design Using $H^{\infty}$ Methods. London: Springer-Verlag, 2000.

[13] A. V. Savkin and I. R. Petersen, "Minimax optimal control of uncertain systems with structured uncertainty," International Journal of Robust and Nonlinear Control, vol. 5, no. 2, pp. 119-137, 1995.

[14] L. Li, V. Ugrinovskii, and R. Orsi, "Decentralized robust control of uncertain Markov jump parameter systems via output feedback," Automatica, 2007. (to appear). 